\title{
Identification of P1 types and variants of Mycoplasma pneumoniae during an epidemic in Chile
}

\section{Correspondence \\ María A. Martínez \\ mamartin@med.uchile.cl}

Received 17 December 2009

Accepted 30 April 2010

\author{
María A. Martínez, ${ }^{1}$ Mauricio Ruiz, ${ }^{2}$ Enna Zunino, ${ }^{3}$ Vivian Luchsinger, ${ }^{4}$ \\ Raúl Aguirre ${ }^{5}$ and Luis F. Avendaño ${ }^{4}$ \\ ${ }^{1}$ Programa de Microbiología, Facultad de Medicina, Universidad de Chile, Santiago de Chile, Chile \\ ${ }^{2}$ Hospital Clínico Universidad de Chile, Santiago de Chile, Chile \\ ${ }^{3}$ Hospital de Infecciosos Dr Lucio Córdova, Santiago de Chile, Chile \\ ${ }^{4}$ Programa de Virología, Facultad de Medicina, Universidad de Chile, Santiago de Chile, Chile \\ ${ }^{5}$ Centro de Síntesis y Análisis de Biomoléculas, Facultad de Medicina, Universidad de Chile, \\ Santiago de Chile, Chile
}

\begin{abstract}
This study was conducted to determine the types of M. pneumoniae prevalent in adults presenting with community-acquired pneumonia during an epidemic period, and to scrutinize a variable region of the RepMP4 element for the detection of P1 variants. All 23 clinical specimens PCRpositive for M. pneumoniae obtained in two hospitals in Santiago, Chile, from 2005 to 2006 were typed by a multiplex PCR directly and then the RepMP4 fragment of 18 specimens was sequenced. A predominance of $M$. pneumoniae type 2 was found, 18 (78.3\%) specimens being grouped as type 2 and $5(21.7 \%)$ as type 1 . Co-infection of $M$. pneumoniae with other respiratory pathogens was found in $10 / 23(43.4 \%)$ patients, but their frequency was not related to the $M$. pneumoniae type. Sequence analysis revealed a single nucleotide polymorphism, a transition mutation, in $50 \%$ of amplicons belonging to type 1 and in $71.4 \%$ of amplicons of type 2 . The nucleotide changes were synonymous in each P1 variant. In conclusion, during the 2005-2006 epidemic in Santiago, both types of $M$. pneumoniae circulated. Although the analysed area in the RepMP4 was small, we detected the existence of P1 variants in the two types of this organism.
\end{abstract}

\section{INTRODUCTION}

Mycoplasma pneumoniae is an important worldwide cause of community-acquired pneumonia (CAP), especially in children and young adults (Waites \& Talkington, 2004). Mycoplasma infections display a cyclical pattern characterized by $4-6$ year endemic periods, alternating with shorter epidemic cycles (Foy et al., 1979; Sasaki et al., 1996; Lind et al., 1997; Layani-Milon et al., 1999). During epidemics, M. pneumoniae is responsible for a significant proportion of CAP cases in all age groups (Layani-Milon et al., 1999). The genome of M. pneumoniae is regarded as genetically stable (Ursi et al., 1994; Dumke et al., 2004; Spuesens et al., 2009). Strains are classified in two main groups (types 1 and 2), which differ in the sequence of two repetitive sequences: RepMP2/3 and RepMP4 found in one copy inside the $\mathrm{P} 1$ gene and in eight to ten copies throughout

Abbreviations: CAP, community-acquired pneumonia; SNP, single nucleotide polymorphism.

The GenBank/EMBL/DDBJ accession numbers for the RepMP4 sequences of the amplicons are G0916909-G0.91625 and G0332741. the chromosome (Ruland et al., 1990; Su et al., 1990; Ursi et al., 1994; Sasaki et al., 1996; Dorigo-Zetsma et al., 2000). Two evolutionary lines (type 1 and 2) of M. pneumoniae exist, and the differences between these lines are reflected in each RepMP2/3 and RepMP4 element within the genome (Spuesens et al., 2009). Studies from Asia, Europe and the USA have reported variations in the distribution of M. pneumoniae types over time, forming the hypothesis that epidemics may arise due to a type shift phenomenon (Ursi et al., 1994; Sasaki et al., 1996; Dumke et al., 2004; Pereyre, et al., 2007; Kenri et al., 2008). Furthermore, several groups have reported the existence of $\mathrm{P} 1$ variants in both M. pneumoniae types (Ruland et al., 1990; Kenri et al., 1999; Dorigo-Zetsma et al., 2001; Pereyre et al., 2007; Spuesens et al., 2009). All described P1 variants have originated by homologous recombination between the P1 RepMP2/3 or RepMP4 repetitive elements (RepMPs) and their extragenic versions (Spuesens et al., 2009). Since P1 protein is a major adhesin and an immunodominant antigen, recombination may serve as a mechanism for P1 variation contributing to the evasion of the immune response of the host (Feldner et al., 1982; Leith et al., 1983). 
The aims of this study were to determine the types of $M$. pneumoniae in specimens collected from adults presenting with CAP during an epidemic period and to scrutinize a variable region of the RepMP4 element for the detection of P1 variants.

\section{METHODS}

Clinical specimens. The 23 specimens characterized in this study correspond to all M. pneumoniae-positive specimens collected during a prospective protocol designed to determine the aetiology of CAP in adults. Patients were recruited at two hospitals in Santiago, Chile, between February 2005 and January 2008 (Martínez et al., 2008). All patients had symptoms suggestive of a lower respiratory tract infection, a new infiltrate on the chest radiograph and no alternative diagnosis emerging during follow-up. All were immunocompetent, had not received immunosuppressive therapy, and had not been hospitalized in the previous 30 days. Positive specimens were obtained between April 2005 and March 2006, a period which coincided with an epidemic of M. pneumoniae in Santiago (Fig. 1). Patients positive for $M$. pneumoniae had a median age of 45 years (range 18-87 years), not
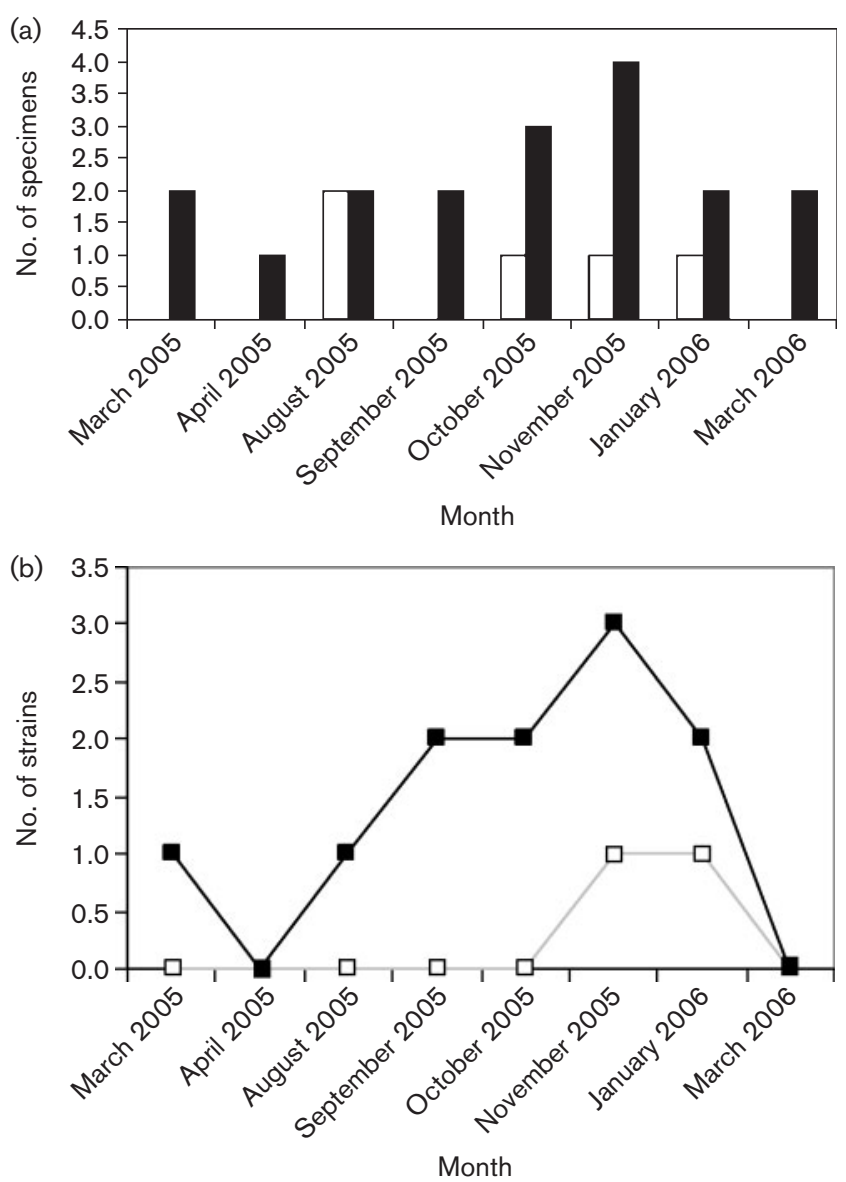

Fig. 1. Distribution of types and variants of the M. pneumoniae P1 gene in clinical specimens collected in Santiago, Chile, between March 2005 and March 2006. (a) Number of specimens of each type detected per month. $\square$, Type $1 ; \mathbf{\square}$, type 2. (b) Number of P1 variants detected per month. $\square$, Type $1 ; \boldsymbol{\square}$, type 2 . significantly different to the median age (65 years) of M. pneumoniaenegative patients $(P=0.1)$ (Martínez et al., 2008).

Microbiological study. The microbiological records for positive $M$. pneumoniae cases from the protocol database were reviewed. The following specimens were obtained for the study: blood, sputum, throat washes, paired sera and urine. Throat washes were processed for indirect immunofluorescent assay (IFA) and cell culture for virus detection, Legionella isolation and for PCR. Streptococcus pneumoniae was diagnosed by conventional blood and sputum cultures and by detection of urinary antigens (BinaxNOW). Chlamydia pneumoniae was tested by nested PCR and serological testing in paired sera (MIF; Savyon) (Martínez et al., 2000). Legionella pneumophila was studied by culture, PCR and detection of urinary antigen (BinaxNOW) (Murdoch, 2003).

IFAs were run for the respiratory viruses influenza $A$ and $B$, respiratory syncytial virus (RSV), adenovirus and parainfluenza viruses (PIV 1-3) using commercial monoclonal antibodies (Chemicon) both for detection as well as for the typing of influenza virus and PIV 1-3. Each specimen was also inoculated into Hep-2 and MDCK cell cultures as described elsewhere (Avendaño et al., 2003). RSV and human metapneumovirus (hMPV) were also detected by real-time PCR assays (Avendaño et al., 2003; Cote et al., 2003).

P1 gene typing. The type of $M$. pneumoniae was determined by the amplification of a variable region of the RepMP4 element of the P1 gene by a multiplex PCR as described by Kenri et al. (2008) with two modifications. Annealing steps were carried out at $58{ }^{\circ} \mathrm{C}$ instead of $55^{\circ} \mathrm{C}$, and Taq DNA polymerase (GoTaq Flexi; Promega) was used in our study. DNA was extracted from throat wash specimens with the Wizard SV Genomic kit (Promega), according to the manufacturer's instructions. M. pneumoniae strain M129 (P1 type 1) and MAC (P1 type 2) were used as reference for comparisons. PCR products consisted of a $343 \mathrm{bp}$ fragment for M. pneumoniae type 1 and a $560 \mathrm{bp}$ fragment for M. pneumoniae type 2.

RepMP4 sequencing. Amplification products of the multiplex PCR of 18 specimens were purified using the QIAquick kit (Qiagen) and sequenced. Sequencing reactions were performed applying the Sequencing Terminator kit (Amersham) in the DNA sequencer ABI 310 (Applied Biosystem). Both strands were sequenced using the forward and reverse primers used in the multiplex PCRs. Sequences were analysed using the BLAST program available from the National Center for Biotechnology Information database (http://www.ncbi. nlm.nih.gov). The GenBank accession numbers for the RepMP4 sequences of the amplicons are GQ916909-GQ91625 and GQ332741.

\section{RESULTS AND DISCUSSION}

\section{M. pneumoniae types}

All 23 specimens positive for M. pneumoniae could be unequivocally assigned to a type. Five specimens $(21.7 \%)$ were grouped as type 1 and 18 specimens $(78.3 \%)$ as type 2 (Fig. 1a). M. pneumoniae infections are historically characterized by a cyclical pattern of epidemics. The distribution of $M$. pneumoniae types during epidemics has shown that in some outbreaks there was a clear predominance of one type over the other, while in other outbreaks, as in our case, both types co-circulated in the population (Sasaki et al., 1996; Dumke et al., 2006; Pereyre et al., 2007; Kenri et al., 2008). By the time that this study was conducted, a prolonged co-circulation of both M. pneumoniae types was observed in Europe, while in Japan most isolates belonged to type 1 
(Dumke et al., 2006; Pereyre et al., 2007; Kenri et al., 2008). Kenri et al. (2008) have suggested that the type shift phenomenon and the spread in the population of a dominant type may be causally linked to epidemic outbreaks. The humoral immune response of patients infected with $M$. pneumoniae, like that of experimentally infected guinea pigs, shows evidence of a protective status directed to the M. pneumoniae type (Jacobs et al., 1996; Dumke et al., 2004). This is not surprising given the key role of the P1 adhesin in the adherence and colonization of the organism in the respiratory tract (Feldner et al., 1982; Leith et al., 1983). Thus, the type shift phenomenon could allow the organism to escape the host immune response, giving rise to an accumulation of susceptible individuals in the population.

Mixed infection was found in 10/23 (43.4\%) patients positive for M. pneumoniae, and presented with a similar frequency in CAP cases caused by type 1 and type 2 organisms. Thus, 2/5 (40\%) co-infections were seen with $M$. pneumoniae type 1 and 8/18 (44.4\%) co-infections occurred with $M$. pneumoniae type 2 . The agents detected were hMPV $(n=4)$, RSV $(n=3)$, C. pneumoniae, S. pneumoniae and L. pneumophila, one in each case. Respiratory viruses are common in adults with pneumonia (Johnstone et al., 2008). Nevertheless, it is difficult to establish the contribution of hMPV and RSV to M. pneumoniae pneumonia cases in our study since they were diagnosed in non-invasive respiratory samples. The use of serological methods may help to clarify a possible role as co-pathogens. hMPV cases clustered in a month during 2006, coincidental with a hMPV epidemic in the city, while RSV infections were scattered throughout the M. pneumoniae circulation period. In six out of seven co-infections with respiratory viruses, IgM, IgG or IgM/IgG seroconversions to $M$. pneumoniae were established. Thus, M. pneumoniae pneumonia probably predisposed to a viral infection, especially when hMPV peaked. Viral infections affected both outpatients $(n=4)$ and hospitalized patients $(n=3)$. C. pneumoniae infection was documented by PCR, and by a decrease in two dilutions of the $\operatorname{IgG}$ titres in the convalescent serum taken 6 weeks apart, while the M. pneumoniae aetiological role is supported by IgG seroconversion. Thus, in this case, C. pneumoniae infection probably preceded and predisposed to a $M$. pneumoniae infection. In the three other mixed infections ( $S$. pneumoniae, L. pneumophila and hMPV), the participation of $M$. pneumoniae was documented only by PCR, as no IgM was detected in the acute phase serum, and no convalescent serum was available. Since $S$. pneumoniae was isolated from the blood cultures, there is no doubt about its involvement, but L. pneumophila was detected only by PCR, which suggests the possibility of a transient patient colonization or a specimen contamination.

\section{RepMP4 polymorphism}

A fragment of the RepMP4 of four $(80 \%)$ specimens belonging to $M$. pneumoniae type 1 and 14 (77.8\%) specimens belonging to type 2 was reamplified and sequenced. The specimens were randomly selected for sequencing; approximately $80 \%$ of each $\mathrm{P} 1$ type specimens were sequenced. Sequence analysis revealed a single nucleotide polymorphism (SNP) in 50\% of amplicons belonging to $\mathrm{P} 1$ type 1 and in $71.4 \%$ amplicons of $\mathrm{P} 1$ type 2. The nucleotide changes corresponded to silent mutations: the transition $573 \mathrm{G} \rightarrow \mathrm{A}$ for type 1 specimens and the transition $891 \mathrm{G} \rightarrow \mathrm{A}$ for type 2 specimens. Only one amplicon, corresponding to a type 2 specimen (accession no. GQ916910), presented a non-silent mutation $(304 \mathrm{G} \longrightarrow \mathrm{T})$, and resulted in the amino acid change $\mathrm{R} \rightarrow \mathrm{L}$ (Table 1). Fig. 1(b) shows the temporal pattern of distribution of $\mathrm{P} 1$ variants. Type 2 variants predominated and were distributed over time, while type 1 variants appeared only twice in a 3 month period. We could not determine differences in the distribution of types and variants by site of collection, since most positive specimens were obtained in one hospital (University Hospital).

Repeated sequences constitute about $8 \%$ of the $M$. pneumoniae genome and as in other bacteria provide DNA for homologous recombination. The RepMP4 element is found in the $5^{\prime}$ part of the P1 gene and also in other seven versions throughout the genome of $M$. pneumoniae. Most nucleotide variation, represented by deletion and insertion events and SNPs, is found in the middle part of the RepMP4 element located in the P1 gene, and allows the differentiation of $M$. pneumoniae in types, but also the recognition of type-independent variants (Spuesens et al., 2009). The SNPs found in this study were similarly located. Since we sequenced only a short extension of the RepMP4, we cannot determine the degree of sequence variation of RepMP4 in our strains.

The identification of SNPs is of interest for the recognition of particular phenotypes that may confer adaptive advantages, e.g. increased virulence, and also for the identification of strains in the community. The SNPs identified in this study will contribute to the knowledge of the epidemiology of M. pneumoniae in our country, since

Table 1. Nucleotide changes found in the RepMP4 sequence of the $\mathrm{P} 1$ gene of 18 specimens positive for M. pneumoniae

Nucleotide changes indicate the variation found in our specimens as compared to reference M. pneumoniae strain M129 (P1 type 1) and MAC (P1 type 2) in GenBank.

\begin{tabular}{|c|c|c|c|}
\hline $\begin{array}{l}\text { P1 } \\
\text { adhesin } \\
\text { type }\end{array}$ & $\begin{array}{l}\text { No. of specimens with } \\
\text { nucleotide changes/ } \\
\text { number of specimens } \\
\text { (\% with changes) }\end{array}$ & $\begin{array}{c}\text { Position of } \\
\text { nucleotide } \\
\text { change }\end{array}$ & $\begin{array}{c}\text { Amino } \\
\text { acid } \\
\text { change }\end{array}$ \\
\hline 1 & $2 / 4(50)$ & $573 \mathrm{G} \longrightarrow \mathrm{A}$ & Silent \\
\hline 2 & $10 / 14(71.4)$ & $891 \mathrm{G} \longrightarrow \mathrm{A}$ & Silent \\
\hline 2 & $1 / 14(7.1)$ & $304 \mathrm{G} \longrightarrow \mathrm{T}$ & $\mathrm{Arg} \rightarrow \mathrm{Leu}$ \\
\hline
\end{tabular}


they will be used as genetic markers to distinguish circulating strains.

One of the limitations of this study is that we did not use a proofreading enzyme, and it is known that conventional DNA Taq polymerase can introduce mutations on amplicons. Furthermore, the genotyping procedure that we used included several rounds of amplifications, increasing the risk of the introduction of mutations. Nevertheless, the finding of identical mutations in several strains in repeated sequencing cycles performed in the variants supports the existence of SNPs in our specimens. Moreover, with a unique exception, transitions involved purines and not pyrimidines as is favoured by DNA Taq polymerase.

To our knowledge, this is the first study that genotypes strains of M. pneumoniae in Latin America. Traditional methods of M. pneumoniae typing required the isolation of the micro-organism, a procedure which is difficult and not sensitive. Later procedures, like those used in our study, allow the typing of the organism directly from clinical specimens by conventional or nested PCR (Dumke et al., 2006; Kenri et al., 2008). However, recently Schwartz et al. (2009a, b) described a combination of real-time PCR and high-resolution analysis of melting curves (HRM) to distinguish between P1 types and to identify variants in isolates of $M$. pneumoniae. In comparison with the procedure used in our study, HRM analysis is faster and easier to perform, and since it does not need further amplicon manipulation, has a lower potential for cross contamination.

In conclusion, during the 2005-2006 epidemic in Santiago, both types of $M$. pneumoniae circulated in the population. Each type exhibited an SNP in RepMP4, a transition mutation that was found in several specimens.

\section{ACKNOWLEDGEMENTS}

Grants FONDECYT-1050735 and FONIS SA 04 i2084 funded an aetiological study of community-acquired pneumonia allowing the collection of strains of $M$. pneumoniae.

\section{REFERENCES}

Avendaño, L. F., Palomino, M. A. \& Larrañaga, C. (2003). Surveillance for respiratory syncytial virus in infants hospitalized for acute lower respiratory infection in Chile (1989 to 2000). J Clin Microbiol 41, 4879-4882.

Cote, S., Abed, Y. \& Boivin, G. (2003). Comparative evaluation of real-time PCR assays for detection of the human metapneumovirus. J Clin Microbiol 41, 3631-3635.

Dorigo-Zetsma, J. W., Dankert, J. \& Zaat, S. A. (2000). Genotyping of Mycoplasma pneumoniae clinical isolates reveals eight P1 subtypes within two genomic groups. J Clin Microbiol 38, 965-970.

Dorigo-Zetsma, J. W., Wilbrink, B., Dankert, J. \& Zaat, S. A. (2001). Mycoplasma pneumoniae P1 type 1- and type 2-specific sequences within the P1 cytadhesin gene of individual strains. Infect Immun 69, $5612-5618$
Dumke, R., Catrein, I., Herrmann, R. \& Jacobs, E. (2004). Preference, adaptation and survival of Mycoplasma pneumoniae subtypes in an animal model. Int J Med Microbiol 294, 149-155.

Dumke, R., Lück, P. C., Noppen, C., Schaefer, C., Von Baum, H., Marre, R. \& Jacobs, E. (2006). Culture-independent molecular subtyping of Mycoplasma pneumoniae in clinical samples. J Clin Microbiol 44, 2567-2570.

Feldner, J., Göbel, U. \& Bredt, W. (1982). Mycoplasma pneumoniae adhesin localized to tip structure by monoclonal antibody. Nature 298, 765-767.

Foy, H. M., Kenny, G. E., Cooney, M. K. \& Allan, I. D. (1979). Longterm epidemiology of infections with Mycoplasma pneumoniae. J Infect Dis 139, 681-687.

Jacobs, E., Vonski, M., Oberle, K., Opitz, O. \& Pietsch, K. (1996). Are outbreaks and sporadic respiratory infections by Mycoplasma pneumoniae due to two distinct subtypes? Eur J Clin Microbiol Infect Dis 15, 38-44.

Johnstone, J., Majumdar, S. R., Fox, J. D. \& Marrie, T. J. (2008). Viral infection in adults hospitalized with community-acquired pneumonia. Prevalence, pathogens, and presentation. Chest 134, 11411148.

Kenri, T., Taniguchi, R., Sasaki, Y., Okazaki, N., Narita, M., Izumikawa, K., Umetsu, M. \& Sasaki, T. (1999). Identification of a new variable sequence in the P1 cytadhesin gene of Mycoplasma pneumoniae: evidence for the generation of antigenic variation by DNA recombination between repetitive sequences. Infect Immun 67, 4557-4562.

Kenri, T., Okazaki, N., Yamazaki, T., Narita, M., Izumikawa, K., Matsuoka, M., Suzuki, S., Horino, A. \& Sasaki, T. (2008). Genotyping analysis of Mycoplasma pneumoniae clinical strains in Japan between 1995 and 2005: type shift phenomenon of M. pneumoniae clinical strains. J Med Microbiol 57, 469-475.

Layani-Milon, M.-P., Gras, I., Valette, M., Luciani, J., Stagnara, J., Aymard, M. \& Lina, B. (1999). Incidence of upper respiratory tract Mycoplasma pneumoniae infections among outpatients in RhoneAlpes, France, during five successive winter periods. J Clin Microbiol 37, 1721-1726.

Leith, D. K., Trevino, L. B., Tully, J. G., Senterfit, L. B. \& Baseman, J. B. (1983). Host discrimination of Mycoplasma pneumoniae proteinaceous immunogens. J Exp Med 157, 502-514.

Lind, K., Benzon, M. W., Jensen, J. S. \& Clyde, W., Jr (1997). A seroepidemiological study of Mycoplasma pneumoniae infections in Denmark over the 50-year period 1946-1995. Eur J Epidemiol 13, 581-586.

Martínez, M. A., Kogan, R., Rojas, P., Rubilar, L., Vidal, R. \& Payá, E. (2000). Diagnosis of Chlamydia pneumoniae in community acquired pneumonia in children in Chile. Acta Paediatr 89, 650-653.

Martínez, M. A., Ruiz, M., Zunino, E., Luchsinger, V. \& Avendaño, L. F. (2008). Detection of Mycoplasma pneumoniae in adult communityacquired pneumonia by PCR and serology. J Med Microbiol 57, 14911495.

Murdoch, D. R. (2003). Diagnosis of Legionella infection. Clin Infect Dis 36, 64-69.

Pereyre, S., Charron, A., Renaudin, H., Bébéar, C. \& Bébéar, C. M. (2007). First report of macrolide-resistant strains and description of a novel nucleotide sequence variation in the P1 adhesin gene in Mycoplasma pneumoniae clinical strains isolated in France over 12 years. J Clin Microbiol 45, 3534-3539.

Ruland, K., Wenzel, R. \& Herrmann, R. (1990). Analysis of three different repeated DNA elements present in the P1 operon of Mycoplasma pneumoniae: size, number and distribution on the genome. Nucleic Acids Res 18, 6311-6317. 
Sasaki, T., Kenry, T., Okazaki, N., Iseki, M., Yamashita, R., Shintani, M., Sasaki, Y. \& Yayoshi, M. (1996). Epidemiological study of Mycoplasma pneumoniae infections in Japan based on PCR-restriction fragment length polymorphism of the P1 cytadhesin gene. J Clin Microbiol 34, 447-449.

Schwartz, S. B., Thurman, K. A., Mitchell, S. L., Wolff, B. J. \& Winchell, J. M. (2009a). Genotyping of Mycoplasma pneumoniae isolates during real-time PCR and high-resolution melt analysis. Clin Microbiol Infect 15, 756-762.

Schwartz, S. B., Mitchell, S. L., Thurman, K. A., Wolff, B. J. \& Winchell, J. M. (2009b). Identification of P1 variants of Mycoplasma pneumoniae by use of high-resolution melt analysis. J Clin Microbiol 47, 41174120.
Spuesens, E. B., Oduber, M., Hoogenboezem, T., Sluijter, M., Hartwig, N. G., Van Rossum, A. M. \& Vink, C. (2009). Sequence variations in RepMP2/3 and RepMP4 elements reveal intragenomic homologous DNA recombination events in Mycoplasma pneumoniae. Microbiology 155, 2182-2196.

Su, C. J., Dallo, S. F. \& Baseman, J. B. (1990). Molecular distinctions among clinical isolates of Mycoplasma pneumoniae. J Clin Microbiol 28, 1538-1540.

Ursi, D., leven, M., Van Bever, H., Quint, W., Niesters, H. G. \& Goossens, H. (1994). Typing of Mycoplasma pneumoniae by PCRmediated DNA fingerprinting. J Clin Microbiol 32, 2873-2875.

Waites, K. B. \& Talkington, D. F. (2004). Mycoplasma pneumoniae and its role as a human pathogen. Clin Microbiol Rev 17, 697-728. 